PROCEEDINGS OF THE

AMERICAN MATHEMATICAL SOCIETY

Volume 138, Number 6, June 2010, Pages 2221-2233

S 0002-9939(10)10237-8

Article electronically published on January 29, 2010

\title{
INTEGER-VALUED POLYNOMIALS AND $K$-THEORY OPERATIONS
}

\author{
M-J. STRONG AND SARAH WHITEHOUSE
}

(Communicated by Brooke Shipley)

\begin{abstract}
This paper provides a unifying approach to recent results linking the fields of integer-valued polynomials and operations in $K$-theory. Following work of Bhargava, we set up a general framework encompassing several examples of rings of integer-valued polynomials. Our main results give bases for the duals of these rings. The rings and their duals all arise in topology as various kinds of cooperations and operations in complex $K$-theory. We show how several previously understood examples fit into this framework and we present some new examples.
\end{abstract}

\section{INTRODUCTION}

Rings of integer-valued polynomials and their duals arise in topology as various kinds of cooperations and operations in complex $K$-theory. Results of this sort can be found in $8,13,9,10$. We present a unifying framework encompassing these results and we also give some new examples. Our main results are Theorems 3.3 and 5.2, giving (topological) bases for the duals of rings of integer-valued polynomials and Laurent polynomials.

To explain further, we introduce some notation. Let $S$ be a subset of $\mathbb{Z}$. We denote by $\operatorname{Int}(S, \mathbb{Z})$ the ring of rational polynomials which take integer values on all elements of $S$,

$$
\operatorname{Int}(S, \mathbb{Z})=\{f(w) \in \mathbb{Q}[w] \mid f(S) \subseteq \mathbb{Z}\} .
$$

Most of our examples are $p$-local. We denote by $\mathbb{Z}_{(p)}$ the $p$-local integers,

$$
\mathbb{Z}_{(p)}=\left\{\frac{a}{b} \in \mathbb{Q} \mid(p, b)=1\right\},
$$

and we consider rings of $p$-locally integer-valued polynomials

$$
\operatorname{Int}\left(S, \mathbb{Z}_{(p)}\right)=\left\{f(w) \in \mathbb{Q}[w] \mid f(S) \subseteq \mathbb{Z}_{(p)}\right\},
$$

for $S$ a subset of $\mathbb{Z}_{(p)}$.

Bhargava 3 gives a way of specifying a regular basis for such a ring of integervalued polynomials. A nice exposition of this work can be found in 4 . In Section 2 we recall his results. We also explain here how, if the subset $S$ of $\mathbb{Z}$ or $\mathbb{Z}_{(p)}$ is closed under multiplication, it follows that the integer-valued polynomial ring is actually a

Received by the editors May 5, 2009, and, in revised form, September 9, 2009.

2010 Mathematics Subject Classification. Primary 55S25; Secondary 13F20, 11B73.

Key words and phrases. K-theory, cohomology operations, integer-valued polynomials.

(C)2010 American Mathematical Society

Reverts to public domain 28 years from publication 
bialgebra, with respect to the comultiplication determined by the polynomial generator being group-like. The duals of these bialgebras of integer-valued polynomials are therefore topological bialgebras.

In Section 3 we consider these duals. Our first main result is Theorem 3.3 giving the dual basis to Bhargava's. We write our dual basis elements as linear combinations of evaluation maps and we call these Adams operations because of the link with $K$-theory operations.

Section 4 presents several examples arising as the cohomology operations in various flavours of complex $K$-theory. Some of these are known examples and we show how they fit into our framework. Theorems 4.1 and 4.2 cover two cases of topological interest where the bases we give are new. The first of these provides a topological basis for the additive unstable bidegree $(0,0)$ operations in $p$-local complex $K$-theory, where the basis elements are polynomials in $\Psi^{p}$ and $\Psi^{q}$ (and $\Psi^{0}$ ). Here $p$ is an odd prime and $q$ is primitive modulo $p^{2}$ (and so a topological generator of the $p$-adic units). The second new example is the split version of this, describing the corresponding operations in the Adams summand of complex $K$-theory. The fact that all such operations are expressible in terms of Adams operations is used in 15 to explore the relationship between these operations and those for complex cobordism.

In Section 5 we consider rings of integer-valued Laurent polynomials. We note how to shift the bases discussed earlier to give bases for these. Theorem 5.2 is our second main result, giving bases for the duals. We explain how various sorts of periodic complex $K$-theory operations fit into this framework.

We end the paper with a table giving a summary of our examples.

This paper is based on work in the Ph.D. thesis of the first author [14], produced under the supervision of the second author.

We would like to thank Francis Clarke for his very helpful comments on a draft version of this paper. Further thanks go to an anonymous referee for comments that led to substantial improvements, in particular to the proofs of the main results, Theorems 3.3 and 5.2 .

\section{General framework}

We begin by setting up some notation which will be useful for describing bases for various subrings of the rational polynomial ring $\mathbb{Q}[w]$. Then we recall some definitions and results from Bhargava [4].

Definition 2.1. Given an integer sequence

$$
\alpha=\left(\alpha_{0}, \alpha_{1}, \alpha_{2}, \alpha_{3}, \ldots\right) \text { with } \alpha_{i} \neq \alpha_{j} \text { for } i \neq j,
$$

we associate to $\alpha$ a sequence of polynomials in $\mathbb{Q}[w]$ by defining, for $n \geq 0$,

$$
f_{n}^{\alpha}(w)=\prod_{i=0}^{n-1} \frac{\left(w-\alpha_{i}\right)}{\left(\alpha_{n}-\alpha_{i}\right)} .
$$

We write

$$
N_{n}^{\alpha}(w)=\prod_{i=0}^{n-1}\left(w-\alpha_{i}\right) \text { and } d_{n}^{\alpha}=\prod_{i=0}^{n-1}\left(\alpha_{n}-\alpha_{i}\right), \text { so that } f_{n}^{\alpha}(w)=\frac{N_{n}^{\alpha}(w)}{d_{n}^{\alpha}} .
$$


Definition 2.2 (Bhargava [4). Let $S \subseteq \mathbb{Z}_{(p)}$. A sequence of elements $\alpha=\left(\alpha_{i}\right)_{i \geq 0}$ of $S$ is a $p$-ordering for $S$ if for all $n \geq 1, \alpha_{n} \in S$ is such that

$$
\nu_{p}\left(d_{n}^{\alpha}\right)=\min \left\{\nu_{p}\left(\prod_{i=0}^{n-1}\left(s-\alpha_{i}\right)\right) \mid s \in S\right\} .
$$

Here $\nu_{p}$ denotes the $p$-adic valuation. The associated $p$-sequence of $S$ is

$$
\left(p^{\nu_{p}\left(d_{n}^{\alpha}\right)}\right) \text {. }
$$

While a $p$-ordering of a subset $S$ of $\mathbb{Z}_{(p)}$ is not unique, the associated $p$-sequence of $S$ is independent of the choice of $p$-ordering and is unique.

A related notion is that of a very well distributed well ordered sequence with respect to $p$ (VWDWO sequence, for short); see [11 and [7, II.2.1]. As noted in [12, a VWDWO sequence is a $p$-ordering for $\mathbb{Z}$ but the converse does not hold. In [12], Johnson studies some examples related to $K$-theory in terms of VWDWO sequences.

Theorem 2.3 (Bhargava [4]). Suppose $\alpha$ is a p-ordering for $S \subseteq \mathbb{Z}_{(p)}$. Then

$$
\left\{f_{n}^{\alpha}(w) \mid n \geq 0\right\}
$$

is a $\mathbb{Z}_{(p)}$-basis for $\operatorname{Int}\left(S, \mathbb{Z}_{(p)}\right)$.

Now let $R$ be either $\mathbb{Z}$ or $\mathbb{Z}_{(p)}$ and let $S$ be a subset of $R$ that is closed under multiplication. We end this section by explaining how this leads to a bialgebra structure on $\operatorname{Int}(S, R)$ for the examples we are interested in.

This involves considering integer-valued polynomials in two variables. So, for $S, S^{\prime} \subseteq R$, define $\operatorname{Int}\left(S \times S^{\prime}, R\right) \subseteq \mathbb{Q}\left[w, w^{\prime}\right]$ as follows:

$$
\operatorname{Int}\left(S \times S^{\prime}, R\right)=\left\{f\left(w, w^{\prime}\right) \in \mathbb{Q}\left[w, w^{\prime}\right] \mid f\left(s, s^{\prime}\right) \in R \text { for all } s \in S, s^{\prime} \in S^{\prime}\right\} .
$$

Suppose that $S$ is such that $\operatorname{Int}(S, R)$ is $R$-free with a regular $R$-basis, say $\left\{f_{n}(w) \mid n \geq 0\right\}$, where the degree of $f_{n}(w)$ is $n$. (For example, by Theorem 2.3. this is the case if $R=\mathbb{Z}_{(p)}$ and $S$ has a $p$-ordering.) Then an inductive argument shows that $\operatorname{Int}(S \times S, R)$ is $R$-free with $R$-basis $\left\{f_{m}(w) f_{n}\left(w^{\prime}\right) \mid n, m \geq 0\right\}$. In other words, the isomorphism

$$
\mathbb{Q}[w] \otimes \mathbb{Q}\left[w^{\prime}\right] \cong \mathbb{Q}\left[w, w^{\prime}\right]
$$

restricts to an isomorphism

$$
\operatorname{Int}(S, R) \otimes \operatorname{Int}(S, R) \cong \operatorname{Int}(S \times S, R) .
$$

Now consider $\mathbb{Q}[w]$ as a bialgebra with comultiplication $\Delta$ determined by the polynomial generator being group-like, $\Delta(w)=w \otimes w$. Under the above isomorphism this comultiplication corresponds to $\Delta(f)\left(w, w^{\prime}\right)=f\left(w w^{\prime}\right)$. Since we suppose $S$ is closed under multiplication it is clear that the restriction of $\Delta: \mathbb{Q}[w] \rightarrow$ $\mathbb{Q}\left[w, w^{\prime}\right]$ to $\operatorname{Int}(S, R)$ lands in $\operatorname{Int}(S \times S, R) \cong \operatorname{Int}(S, R) \otimes \operatorname{Int}(S, R)$.

With this structure $\operatorname{Int}(S, R)$ is a bialgebra and its $R$-linear dual is a topological bialgebra. 


\section{Duality}

In this section we produce topological bases, expressed in terms of evaluation maps, for the linear duals of the subrings of $\mathbb{Q}[w]$ considered above. Motivated by the link to $K$-theory operations, we refer to these evaluation maps as Adams operations.

Definition 3.1. Let $S \subseteq \mathbb{Z}_{(p)}$ and let $\gamma \in S$. The Adams operation $\Psi^{\gamma}$ is given by evaluation at $\gamma$,

$$
\begin{aligned}
\Psi^{\gamma}: \operatorname{Int}\left(S, \mathbb{Z}_{(p)}\right) & \rightarrow \mathbb{Z}_{(p)} \\
f(w) & \mapsto f(\gamma) .
\end{aligned}
$$

Definition 3.2. For a sequence $\alpha=\left(\alpha_{i}\right)_{i \geq 0}$ of $p$-local integers, let

$$
N_{n, j}^{\alpha}(w)=\prod_{\substack{0 \leq i \leq n-1 \\ i \neq j}}\left(w-\alpha_{i}\right) \quad \in \mathbb{Z}_{(p)}[w] .
$$

We write $\operatorname{Int}\left(S, \mathbb{Z}_{(p)}\right)^{*}$ for the $\mathbb{Z}_{(p)}$-linear dual of $\operatorname{Int}\left(S, \mathbb{Z}_{(p)}\right)$, that is, for

$$
\operatorname{Hom}_{\mathbb{Z}_{(p)}}\left(\operatorname{Int}\left(S, \mathbb{Z}_{(p)}\right), \mathbb{Z}_{(p)}\right) .
$$

Theorem 3.3. Suppose $\alpha=\left(\alpha_{i}\right)_{i \geq 0}$ is a p-ordering for $S \subseteq \mathbb{Z}_{(p)}$. Let

$$
\theta_{n}^{\alpha}=\Psi^{\alpha_{n}}-\sum_{j=0}^{n-1} \frac{N_{n, j}^{\alpha}\left(\alpha_{n}\right)}{N_{n, j}^{\alpha}\left(\alpha_{j}\right)} \Psi^{\alpha_{j}} .
$$

Then $\left\{\theta_{n}^{\alpha} \mid n \geq 0\right\}$ is the topological $\mathbb{Z}_{(p)}$-basis for $\operatorname{Int}\left(S, \mathbb{Z}_{(p)}\right)^{*}$ dual to the $f_{n}^{\alpha}(w)$. Moreover, the coefficients $\frac{N_{n, j}^{\alpha}\left(\alpha_{n}\right)}{N_{n, j}^{\alpha}\left(\alpha_{j}\right)}$ lie in $\mathbb{Z}_{(p)}$.

Proof. To show that the $\theta_{n}^{\alpha}$ constitute the dual basis to the $f_{n}^{\alpha}(w)$ we need to show that

$$
f_{m}^{\alpha}\left(\alpha_{n}\right)-\sum_{j=0}^{n-1} \frac{N_{n, j}^{\alpha}\left(\alpha_{n}\right)}{N_{n, j}^{\alpha}\left(\alpha_{j}\right)} f_{m}^{\alpha}\left(\alpha_{j}\right)=\delta_{n, m}
$$

for all $m, n \geq 0$. Of course, it follows that $\theta_{n}^{\alpha} \in \operatorname{Int}\left(S, \mathbb{Z}_{(p)}\right)^{*}$. Since

$$
f_{m}^{\alpha}\left(\alpha_{j}\right)= \begin{cases}1 & \text { if } j=m, \\ 0 & \text { if } j<m,\end{cases}
$$

it is clear that (3.1) holds for $n \leq m$.

Let $\pi_{n}^{\alpha}: \mathbb{Q}[w] \rightarrow \mathbb{Q}[w]$ be the linear projection that maps a polynomial $g(w)$ to the unique polynomial of degree less than $n$ that takes the same values as $g$ at each of $\alpha_{0}, \alpha_{1}, \ldots, \alpha_{n-1}$. By Lagrange's interpolation formula,

$$
\pi_{n}^{\alpha}(g)(w)=\sum_{j=0}^{n-1} \frac{N_{n, j}^{\alpha}(w)}{N_{n, j}^{\alpha}\left(\alpha_{j}\right)} g\left(\alpha_{j}\right) .
$$

So the left-hand side of (3.1) is the value at $w=\alpha_{n}$ of $f_{m}^{\alpha}(w)-\pi_{n}^{\alpha}\left(f_{m}^{\alpha}\right)(w)$. But $\pi_{n}^{\alpha}$ fixes $f_{m}^{\alpha}(w)$ if $m<n$, since $f_{m}^{\alpha}(w)$ has degree $m$. Hence we have shown that (3.1) holds for all $m, n \geq 0$. 
The formula (3.1) shows that the upper unitriangular matrix whose $(j, n)$-entry, for $j<n$, is $-\frac{N_{n, j}^{\alpha}\left(\alpha_{n}\right)}{N_{n, j}^{\alpha}\left(\alpha_{j}\right)}$ is the inverse of the unitriangular matrix $\left(f_{m}^{\alpha}\left(\alpha_{j}\right)\right)_{m, j \geq 0}$. Since $f_{m}^{\alpha}\left(\alpha_{j}\right) \in \mathbb{Z}_{(p)}$, it follows that $\frac{N_{n, j}^{\alpha}(w)}{N_{n, j}^{\alpha}\left(\alpha_{j}\right)} \in \mathbb{Z}_{(p)}$.

We note that the proof above also shows that

$$
\Psi^{\alpha_{n}}=\sum_{j=0}^{n} f_{j}^{\alpha}\left(\alpha_{n}\right) \theta_{j}^{\alpha}
$$

As discussed at the end of Section 2, if $S$ is multiplicatively closed, $\operatorname{Int}\left(S, \mathbb{Z}_{(p)}\right)$ is a bialgebra and the $\operatorname{dual} \operatorname{Int}\left(S, \mathbb{Z}_{(p)}\right)^{*}$ is a topological bialgebra. For $\beta, \gamma \in S$, the evaluation maps $\Psi^{\beta}$ and $\Psi^{\gamma}$ multiply as $\Psi^{\beta} \Psi^{\gamma}=\Psi^{\beta \gamma}$ (which is indeed how Adams operations compose).

\section{EXAmples RElated to $K$-THEORY Operations}

In this section we present several examples of sequences $\left(\alpha_{i}\right)_{i \geq 0}$, each corresponding via Theorem 2.3 to a basis of an interesting ring of integer-valued polynomials. In each one the set $S$ is closed under multiplication and so we have a comultiplication on the integer-valued polynomials. All of these examples arise as cooperations in various flavours of complex $K$-theory. Thus their duals are interesting rings of operations.

We will use some standard notation and terminology relating to operations and cooperations in a cohomology theory $E$; see [5, 6] for further details. In our examples, $E$ will be either complex $K$-theory, written $K$, sometimes with $p$-local coefficients and then written $K_{(p)}$, or its Adams summand, which we denote by $G$. We also need the corresponding connective versions, written $k, k_{(p)}$ and $g$ respectively.

The degree zero stable cohomology operations for the theory $E$ are given by $E^{0}(E)=[E, E]$. For our examples, this (topological) bialgebra is dual to the bialgebra of cooperations $E_{0}(E)$.

The bidegree $(0,0)$ unstable operations are given by $E^{0}\left(\underline{E}_{0}\right)=\left[\underline{E}_{0}, \underline{E}_{0}\right]$, where $\underline{E}_{0}$ is the zero space of an $\Omega$-spectrum representing $E$. Inside here are the additive unstable bidegree $(0,0)$ operations, denoted by $P E^{0}\left(\underline{E}_{0}\right)$. For our examples, this topological ring is dual to the corresponding additive cooperations $Q E_{0}\left(\underline{E}_{0}\right)$, the indecomposable quotient of $E_{0}\left(\underline{E}_{0}\right)$ with respect to the $\star$-product.

The relevance of rings of integer-valued polynomials to the study of operations in $K$-theory has been understood for a long time. The first example below is classical and is related to additive unstable operations in $K$-theory, as explained in [8, 13. Examples (4.2) and (4.3) relate to stable operations, and these bases were presented in [9, 10]. The bases given in examples (4.4) and (4.5) relate to additive unstable operations, this time in the $p$-local setting, and these are new.

Theorem 3.3 provides formulas for the dual bases. In the first three examples the formula simplifies to give nice coefficients. We do not know any correspondingly nice expression for the coefficients for examples (4.4) and (4.5).

(4.1) We take $S=\mathbb{Z}$, so we are considering the classical ring of integer-valued polynomials $\operatorname{Int}(\mathbb{Z}, \mathbb{Z})$. The ring of additive cooperations for complex $K$ theory $Q K_{0}\left(\underline{K}_{0}\right)$ is isomorphic to $\operatorname{Int}(\mathbb{Z}, \mathbb{Z})$, as explained in [8, 13]. As is 
well known, the binomial coefficient polynomials

$$
\left(\begin{array}{l}
w \\
n
\end{array}\right)=\frac{w(w-1) \ldots(w-n+1)}{n !}
$$

for $n \geq 0$, form a $\mathbb{Z}$-basis. This is a basis of the sort given in Theorem 2.3. with $\alpha_{i}=i$ for $i \geq 0$, where this sequence is a $p$-ordering of $\mathbb{Z}$ for every prime $p$.

The dual $\operatorname{Int}(\mathbb{Z}, \mathbb{Z})^{*}$ is isomorphic to the ring $P K^{0}\left(\underline{K}_{0}\right)$ of additive bidegree $(0,0)$ operations in complex $K$-theory. Indeed such an operation $\theta$ is uniquely determined by $\theta([\eta]) \in K^{0}\left(\mathbb{C} P^{\infty}\right)=\mathbb{Z}[[t]]$, where $t=[\eta-1]$ and $\eta$ is the universal line bundle over $\mathbb{C} P^{\infty}$. The dual topological basis elements to the binomial coefficient polynomials given by Theorem 3.3 are

$$
\sigma_{n}=\sum_{j=0}^{n}(-1)^{n-j}\left(\begin{array}{l}
n \\
j
\end{array}\right) \Psi^{j} .
$$

These elements were used by Adams in [1]. Under the isomorphism

$$
\operatorname{Int}(\mathbb{Z}, \mathbb{Z})^{*} \cong \mathbb{Z}[[t]],
$$

$\sigma_{n}$ corresponds to $t^{n}$. This is an isomorphism of groups. It becomes an isomorphism of rings if one considers the ring structure on $\operatorname{Int}(S, \mathbb{Z})^{*}$ coming from addition on $S=\mathbb{Z}$. However, rather than the usual power series ring structure on $K^{0}\left(\mathbb{C} P^{\infty}\right)$, we are interested in the ring structure on $P K^{0}\left(\underline{K}_{0}\right)$ corresponding to composition of operations. This is determined by the rule $\Psi^{j} \Psi^{l}=\Psi^{j l}$ for composing Adams operations, and as explained earlier this is dual to multiplication on $S=\mathbb{Z}$.

In this example, the combinatorial identity establishing the duality is

$$
\sum_{j}(-1)^{n-j}\left(\begin{array}{c}
n \\
j
\end{array}\right)\left(\begin{array}{c}
j \\
m
\end{array}\right)=\delta_{n, m} .
$$

(4.2) We consider $\operatorname{Int}\left(\mathbb{Z}_{(p)}^{\times}, \mathbb{Z}_{(p)}\right)$ where $\mathbb{Z}_{(p)}^{\times}$denotes the $p$-local units. As explained in [9], this is isomorphic to the ring $K_{(p) 0}\left(k_{(p)}\right)$ of degree zero stable cooperations in $p$-local complex $K$-theory.

Let $p$ be an odd prime and let $q$ be primitive $\bmod p^{2}$. By [9, Proposition 3], there is a $\mathbb{Z}_{(p)}$-basis for $K_{(p) 0}\left(k_{(p)}\right)$ of the sort given by Theorem 2.3, with $\alpha_{i}=q^{i}$, for $i \geq 0$.

For non-negative integers $j$ and $n$, let

$$
\left[\begin{array}{l}
n \\
j
\end{array}\right]_{q}=\prod_{i=0}^{j-1} \frac{1-q^{n-i}}{1-q^{j-i}},
$$

a Gaussian polynomial evaluated at $q$. The dual of $K_{(p) 0}\left(k_{(p)}\right)$ is the ring $k_{(p)}^{0}\left(k_{(p)}\right)$ of stable degree zero operations for connective $p$-local $K$-theory. In this case the dual topological basis given by Theorem 3.3 is

$$
\varphi_{n}=\sum_{j=0}^{n}(-1)^{n-j} q^{\left(\begin{array}{c}
n-j \\
2
\end{array}\right)}\left[\begin{array}{l}
n \\
j
\end{array}\right]_{q} \Psi^{q^{j}}
$$

as in [10, Proposition 2.4]. 
Theorem 2.2 of [10] shows that $\varphi_{n}$ factors as

$$
\varphi_{n}=\prod_{k=0}^{n-1}\left(\Psi^{q}-q^{k}\right), \quad \text { for } n \geq 0 .
$$

The combinatorial identity establishing the duality is

$$
\sum_{j}(-1)^{n-j} q^{\left(\begin{array}{c}
n-j \\
2
\end{array}\right)}\left[\begin{array}{l}
n \\
j
\end{array}\right]_{q}\left[\begin{array}{l}
j \\
m
\end{array}\right]_{q}=\delta_{n, m} .
$$

For $p=2$, things work slightly differently. By [9, Proposition 20], we get a $\mathbb{Z}_{(2)}$-basis of the type given by Theorem 2.3 for $\operatorname{Int}\left(\mathbb{Z}_{(2)}^{\times}, \mathbb{Z}_{(2)}\right) \cong K_{(2)_{0}}\left(k_{(2)}\right)$, with $\alpha_{i}=(-1)^{i} 3^{\lfloor i / 2\rfloor}$, for $i \geq 0$.

(4.3) Let $p$ be an odd prime and let $q$ be primitive $\bmod p^{2}$. We consider

$$
\begin{aligned}
\operatorname{Int}\left(\left(\mathbb{Z}_{(p)}^{\times}\right)^{p-1}, \mathbb{Z}_{(p)}\right) & =\operatorname{Int}\left(1+p \mathbb{Z}_{(p)}, \mathbb{Z}_{(p)}\right) \\
& =\left\{f(z) \in \mathbb{Q}[z] \mid f\left(1+p \mathbb{Z}_{(p)}\right) \subseteq \mathbb{Z}_{(p)}\right\} .
\end{aligned}
$$

(Here the first equality follows from $p$-adic continuity and the fact that $\left(\mathbb{Z}_{p}^{\times}\right)^{p-1}=1+p \mathbb{Z}_{p}$, where $\mathbb{Z}_{p}$ denotes the $p$-adic integers. In the terminology of Cahen-Chabert [7, IV.1.2] the above equality of rings of integervalued polynomials is expressed by saying that $\left(\mathbb{Z}_{(p)}^{\times}\right)^{p-1}$ and $1+p \mathbb{Z}_{(p)}$ are polynomially $\mathbb{Z}_{(p)}$-equivalent.)

As explained in [10, this ring of integer-valued polynomials is isomorphic to $G_{0}(g)$, where $G$ and $g$ are the Adams summands of $p$-local periodic and connective $K$-theory respectively. Note that here, to see $G_{0}(g)$ sitting inside $K_{(p) 0}\left(k_{(p)}\right)$, we should think of the variable $z$ as $w^{p-1}$.

By [10, Proposition 4.2], this has a $\mathbb{Z}_{(p)}$-basis $\left\{f_{n}^{\alpha}(z) \mid n \geq 0\right\}$ of the type given in Theorem 2.3. with $\alpha_{i}=\hat{q}^{i}$ for $i \geq 0$, where $\hat{q}=q^{p-1}$.

The dual of $G_{0}(g)$ is $g^{0}(g)$, the ring of stable degree zero operations for the connective Adams summand. It follows from [10, Theorem 4.4] that the dual topological basis elements $\hat{\varphi}_{n}$ given by Theorem 3.3 factor as

$$
\hat{\varphi}_{n}=\prod_{k=0}^{n-1}\left(\Psi^{q}-\hat{q}^{k}\right), \quad \text { for } n \geq 0 .
$$

The identity establishing the duality is the hatted version of that in the previous example. Notice that we write $\Psi^{q}$ to mean evaluation at $w=q$; equivalently this is evaluation at $z=\hat{q}$.

Again there is a variant for $p=2$. One considers $\operatorname{Int}\left(\left(\mathbb{Z}_{(2)}^{\times}\right)^{2}, \mathbb{Z}_{(2)}\right) \cong$ $K O_{(2)_{0}}\left(k o_{(2)}\right)$, where $K O_{(2)}$ is 2-local real $K$-theory and $k o_{(2)}$ is its connective cover. By [10, Proposition 9.2], a $\mathbb{Z}_{(2)}$-basis for this is given by $\left\{f_{n}^{\alpha}\left(w^{2}\right) \mid n \geq 0\right\}$, where $\alpha_{i}=9^{i}$ for $i \geq 0$.

(4.4) Let $p$ be an odd prime. Here we consider a $\mathbb{Z}_{(p)}$-basis of the ring of $p$-local integer-valued polynomials, $\operatorname{Int}\left(\mathbb{Z}, \mathbb{Z}_{(p)}\right)=\operatorname{Int}\left(\mathbb{Z}_{(p)}, \mathbb{Z}_{(p)}\right)$. It follows from the discussion of example (4.1) that $Q K_{(p)_{0}}\left(\underline{K_{(p)}}{ }_{0}\right)$ is isomorphic to $\operatorname{Int}\left(\mathbb{Z}, \mathbb{Z}_{(p)}\right)$.

Of course, the polynomials $\left(\begin{array}{l}w \\ n\end{array}\right)$, being an integral basis for $\operatorname{Int}(\mathbb{Z}, \mathbb{Z})$, also form a $\mathbb{Z}_{(p)}$-basis for $\operatorname{Int}\left(\mathbb{Z}, \mathbb{Z}_{(p)}\right)$. The advantage of the alternative basis 
given below lies in the fact that it leads to a dual basis of $\operatorname{Int}\left(\mathbb{Z}, \mathbb{Z}_{(p)}\right)^{*}$ involving polynomials in only $\Psi^{0}, \Psi^{p}$ and $\Psi^{q}$, rather than all the $\Psi^{k}$. Also, this formulation leads naturally to example (4.5) below, where a basis for the operations was not previously known.

Theorem 4.1. Let $p$ be an odd prime and let $q$ be primitive $\bmod p^{2}$. Let $\alpha_{0}=0$ and, for $m \geq 1$, let $\alpha_{m}=p^{i} q^{j-1-\lfloor j / p\rfloor}$ where $m=p^{i} j>0$ with $p \nmid j$. Then $\left\{f_{n}^{\alpha}(w) \mid n \geq 0\right\}$ is a $\mathbb{Z}_{(p)}$-basis of the ring of $p$-local integer-valued polynomials, $\operatorname{Int}\left(\mathbb{Z}, \mathbb{Z}_{(p)}\right)$.

Proof. Example (4.1) shows that the $p$-sequence of $\mathbb{Z}$ is $\left(p^{\nu_{p}(k !)}\right)_{k \geq 0}$. We claim that for all $m, n \geq 0$, we have $\nu_{p}\left(\alpha_{m}-\alpha_{n}\right)=\nu_{p}(m-n)$. It follows that $\nu_{p}\left(d_{n}^{\alpha}\right)=\nu_{p}(n !)$ for all $n \geq 1$, and then the result follows from Theorem 2.3.

To prove the claim, first note that it is true if either of $m$ or $n$ is 0 . So suppose $m, n \neq 0$ and let $m=p^{i} j, n=p^{k} l$ where $p \nmid j, l$. Without loss of generality, assume $i \geq k$. Then

$$
\begin{aligned}
\nu_{p}\left(\alpha_{m}-\alpha_{n}\right) & =\nu_{p}\left(p^{i} q^{j-1-\lfloor j / p\rfloor}-p^{k} q^{l-1-\lfloor l / p\rfloor}\right) \\
& = \begin{cases}k+\nu_{p}\left(q^{r}-1\right) & \text { if } i=k, \\
k & \text { if } i \neq k,\end{cases}
\end{aligned}
$$

where $r=j-\lfloor j / p\rfloor-l+\lfloor l / p\rfloor$. This gives

$$
\nu_{p}\left(\alpha_{m}-\alpha_{n}\right)= \begin{cases}k+1+\nu_{p}(r) & \text { if } i=k \text { and } p-1 \text { divides } r \\ k & \text { otherwise. }\end{cases}
$$

On the other hand we have

$$
\begin{aligned}
\nu_{p}(m-n) & =\nu_{p}\left(p^{i} j-p^{k} l\right) \\
& = \begin{cases}k+\nu_{p}(j-l) & \text { if } i=k, \\
k & \text { if } i \neq k .\end{cases}
\end{aligned}
$$

Let $j=p s+t$ and $l=p u+v$ where $t, v \in\{1, \ldots, p-1\}$. Then

$$
j-l=p(s-u)+t-v
$$

and

$$
r=(p-1)(s-u)+t-v .
$$

Suppose $p-1$ divides $r$. Then $p-1$ divides $t-v$, so $t-v=0$ since $t-v \in$ $\{-(p-2), \ldots, p-2\}$. In this case,

$$
\nu_{p}(j-l)=\nu_{p}(p(s-u))=1+\nu_{p}(s-u),
$$

and

$$
1+\nu_{p}(r)=1+\nu_{p}((p-1)(s-u))=1+\nu_{p}(s-u) .
$$

Now suppose $p-1$ does not divide $r$. Then $p-1$ does not divide $t-v$, so $t-v \neq 0$. In this case, $p$ cannot divide $t-v$ since $t-v \in\{-(p-2), \ldots, p-2\}$, so

$$
\nu_{p}(j-l)=\nu_{p}(p(s-u)+t-v)=0 .
$$

(4.5) Let $p$ be an odd prime. We consider

$$
\operatorname{Int}\left(\mathbb{Z}_{(p)}^{p-1}, \mathbb{Z}_{(p)}\right)=\left\{f(z) \in \mathbb{Q}[z] \mid f\left(\mathbb{Z}_{(p)}^{p-1}\right) \subseteq \mathbb{Z}_{(p)}\right\} .
$$


This is isomorphic to the additive cooperations for the Adams summand $Q G_{0}\left(\underline{G}_{0}\right)$, where, as in example (4.3), we should think of the variable $z$ as $w^{p-1}$, in order to see the inclusion

$$
\begin{aligned}
Q G_{0}\left(\underline{G}_{0}\right) & =Q K_{(p)_{0}}\left({\underline{K_{(p)}}}_{0}\right) \cap \mathbb{Q}\left[w^{p-1}\right] \\
& =\left\{f(w) \in \mathbb{Q}[w] \mid f\left(\mathbb{Z}_{(p)}\right) \subseteq \mathbb{Z}_{(p)}\right\} \cap \mathbb{Q}\left[w^{p-1}\right] .
\end{aligned}
$$

It is straightforward to see that one basis of $\operatorname{Int}\left(\mathbb{Z}_{(p)}^{p-1}, \mathbb{Z}_{(p)}\right)$ is given by $\left\{\pi\left(\begin{array}{l}w \\ n\end{array}\right) \mid n \geq 0\right\}$, where $\pi: \mathbb{Q}[w] \rightarrow \mathbb{Q}[w]$ is the $\mathbb{Q}$-linear map given by

$$
\pi\left(w^{i}\right)= \begin{cases}w^{i} & \text { if } p-1 \text { divides } i \\ 0 & \text { otherwise. }\end{cases}
$$

However, this basis is not of the sort we have been considering and we do not know what the dual basis is. We give an alternative basis that fits into our framework.

Theorem 4.2. Let $p$ be an odd prime and let $q$ be primitive $\bmod p^{2}$. Let $\hat{p}=p^{p-1}$ and $\hat{q}=q^{p-1}$. Let $\alpha_{0}=0$ and, for $m \geq 1$, let $\alpha_{m}=\hat{p}^{i} \hat{q}^{j-1-\left\lfloor\frac{j}{p}\right\rfloor}$ where $m=p^{i} j>0$ with $p \nmid j$. Then $\left\{f_{n}^{\alpha}(z) \mid n \geq 0\right\}$ is a $\mathbb{Z}_{(p)}$-basis of the ring $\operatorname{Int}\left(\mathbb{Z}_{(p)}^{p-1}, \mathbb{Z}_{(p)}\right)$.

Proof. It follows from the remarks preceding the theorem that the $p$-sequence of $\mathbb{Z}^{p-1}$ is $\left(p^{\nu_{p}(((p-1) k) !)}\right)_{k \geq 0}$. The result will follow from Theorem 2.3, provided that $\nu_{p}\left(d_{n}^{\alpha}\right)=\nu_{p}(((p-1) n) !)$.

To prove this we introduce some notation. We will write $\rho_{n, i}$ for $\left\lfloor\frac{n-1}{p^{i}}\right\rfloor-\left\lfloor\frac{n-1}{p^{i+1}}\right\rfloor-$ 1 for $i \geq 0, n \in \mathbb{Z}$. Hence, $\alpha_{n}=\hat{p}^{i} \hat{q}^{\rho_{n+1, i}}$ for $n=p^{i} j>0$ with $p \nmid j$, and one can check that

$$
d_{n}^{\alpha}=\alpha_{n} \prod_{l=0}^{r} \prod_{j=0}^{\rho_{n, l}}\left(\alpha_{n}-\hat{p}^{l} \hat{q}^{j}\right)
$$

where $r$ is such that $p^{r} \leq n-1<p^{r+1}$.

Let $0 \leq l \leq r$. We have

$$
\nu_{p}\left(\alpha_{n}-\hat{p}^{l} \hat{q}^{t}\right)= \begin{cases}l(p-1) & \text { if } 0 \leq l<i, \\ i(p-1) & \text { if } l>i, \\ i(p-1)+\nu_{p}\left(\hat{q}^{\rho_{n+1, i}}-\hat{q}^{t}\right) & \text { if } l=i\end{cases}
$$

Thus

$$
\begin{aligned}
\nu_{p}\left(d_{n}^{\alpha}\right) & =n i(p-1)-\sum_{l=0}^{i-1} \sum_{t=0}^{\rho_{n, l}}(i-l)(p-1)+\sum_{j=0}^{\rho_{n, i}} \nu_{p}\left(\hat{q}^{\rho_{n+1, i}}-\hat{q}^{j}\right) \\
& =n i(p-1)-(p-1) \sum_{l=0}^{i-1}(i-l) \frac{n}{p^{l+1}}+\sum_{j=0}^{\rho_{n, i}} \nu_{p}\left(\hat{q}^{\rho_{n+1, i}}-\hat{q}^{j}\right) \\
& =n(p-1) \sum_{l=1}^{i} \frac{1}{p^{l}}+\sum_{j=0}^{\rho_{n, i}} \nu_{p}\left(\hat{q}^{\rho_{n+1, i}}-\hat{q}^{j}\right) .
\end{aligned}
$$

A routine calculation, using the fact that $\nu_{p}\left(\hat{q}^{j}-1\right)=1+\nu_{p}(j)$, shows that

$$
\sum_{j=0}^{\rho_{n, i}} \nu_{p}\left(\hat{q}^{\rho_{n+1, i}}-\hat{q}^{j}\right)=\left\lfloor\frac{(p-1) n}{p^{i+1}}\right\rfloor+\nu_{p}\left(\left(\left\lfloor\frac{(p-1) n}{p^{i+1}}\right\rfloor\right) !\right) .
$$


So

$$
\begin{aligned}
\nu_{p}\left(d_{n}^{\alpha}\right) & =n(p-1) \sum_{l=1}^{i} \frac{1}{p^{l}}+\left\lfloor\frac{(p-1) n}{p^{i+1}}\right\rfloor+\nu_{p}\left(\left(\left\lfloor\frac{(p-1) n}{p^{i+1}}\right\rfloor\right) !\right) \\
& =\sum_{l=1}^{\infty}\left\lfloor\frac{(p-1) n}{p^{l}}\right\rfloor \\
& =\nu_{p}(((p-1) n) !) .
\end{aligned}
$$

A particularly nice feature of examples (4.2) and (4.3) is a kind of "self-duality": up to denominators, the original basis and its dual each consists of the same polynomials in a single variable.

\section{LAURENT POLYNOMIALS}

In this section we extend our results to some rings of Laurent polynomials satisfying integrality conditions. Further examples related to $K$-theory fit into this framework.

We follow the argument of [9, Corollary 6], which in turn comes from [2], to shift a basis for polynomials to one for Laurent polynomials.

Proposition 5.1. Let $\alpha=\left(\alpha_{0}, \alpha_{1}, \alpha_{2}, \ldots\right)$ be a sequence with $\alpha_{i} \neq \alpha_{j}$ for $i \neq j$ and $\alpha_{i} \in \mathbb{Z}_{(p)}^{\times}$for all $i$. Let $R$ be a $\mathbb{Z}_{(p)}\left[w, w^{-1}\right]$-submodule of $\mathbb{Q}\left[w, w^{-1}\right]$. Suppose that $\left\{f_{n}^{\alpha}(w) \mid n \geq 0\right\}$ is a $\mathbb{Z}_{(p)}$-basis for $R \cap \mathbb{Q}[w]$. Then $\left\{w^{-\lfloor n / 2\rfloor} f_{n}^{\alpha}(w) \mid n \geq 0\right\}$ is $a \mathbb{Z}_{(p)}$-basis for $R$.

Proof. For $j \leq k \in \mathbb{Z}$, let $F(j, k)=R \cap \mathbb{Q}\left\{w^{j}, w^{j+1}, \ldots, w^{k}\right\}$. Let $N$ be a positive integer. For each $n=0,1, \ldots, N$, we may write $w^{\lfloor N / 2\rfloor-\lfloor n / 2\rfloor} f_{n}^{\alpha}(w) \in F(0, N)$ as $\sum_{i=0}^{N} x_{n, i} f_{i}^{\alpha}(w)$, where, by duality, $x_{n, i}=\theta_{i}^{\alpha}\left(w^{\lfloor N / 2\rfloor-\lfloor n / 2\rfloor} f_{n}^{\alpha}(w)\right)$, which, by Theorem 3.3, satisfies

$$
x_{n, i}= \begin{cases}0, & \text { if } 0 \leq i<n, \\ \alpha_{n}^{\lfloor N / 2\rfloor-\lfloor n / 2\rfloor}, & \text { if } i=n .\end{cases}
$$

This shows that the $w^{\lfloor N / 2\rfloor-\lfloor n / 2\rfloor} f_{n}^{\alpha}(w)$ form a $\mathbb{Z}_{(p)}$-basis for $F(0, N)$, since the matrix expressing them in terms of the $f_{n}^{\alpha}(w)$ is upper-triangular, with units on the diagonal, and hence invertible over $\mathbb{Z}_{(p)}$. Thus

$$
\left\{w^{-\lfloor n / 2\rfloor} f_{n}^{\alpha}(w) \mid 0 \leq n \leq N\right\}
$$

forms a basis for $F(-\lfloor N / 2\rfloor,\lceil N / 2\rceil)$, and the result follows.

Now we give the dual to this shifted basis. Once again the basis elements are linear combinations of Adams operations (that is, evaluation maps). Note that since we assume $\alpha_{j} \in \mathbb{Z}_{(p)}^{\times}$, it follows from Proposition [5.1 that $\Psi^{\alpha_{j}} \in R^{*}=$ $\operatorname{Hom}_{\mathbb{Z}_{(p)}}\left(R, \mathbb{Z}_{(p)}\right)$. Our dual basis takes a very similar form to that of the dual to the original unshifted basis.

Theorem 5.2. Let $\alpha$ and $R$ be as in Proposition 5.1. Let

$$
\Theta_{n}^{\alpha}=\alpha_{n}^{\lfloor n / 2\rfloor} \Psi^{\alpha_{n}}-\alpha_{n}^{\epsilon} \sum_{j=0}^{n-1} \alpha_{j}^{\lfloor(n-1) / 2\rfloor} \frac{N_{n, j}^{\alpha}\left(\alpha_{n}\right)}{N_{n, j}^{\alpha}\left(\alpha_{j}\right)} \Psi^{\alpha_{j}} \quad \in R^{*},
$$


where $\epsilon \in\{0,1\}$ is such that $\epsilon \equiv n-1(\bmod 2)$. Then $\left\{\Theta_{n}^{\alpha} \mid n \geq 0\right\}$ is the (topological) dual basis to the $w^{-\lfloor n / 2\rfloor} f_{n}^{\alpha}(w)$.

Proof. We need to show that

$$
\alpha_{n}^{\lfloor n / 2\rfloor-\lfloor m / 2\rfloor} f_{m}^{\alpha}\left(\alpha_{n}\right)-\alpha_{n}^{\epsilon} \sum_{j=0}^{n-1} \alpha_{j}^{\lfloor(n-1) / 2\rfloor-\lfloor m / 2\rfloor} \frac{N_{n, j}^{\alpha}\left(\alpha_{n}\right)}{N_{n, j}^{\alpha}\left(\alpha_{j}\right)} f_{m}^{\alpha}\left(\alpha_{j}\right)=\delta_{n, m},
$$

for all $m, n \geq 0$.

As in the proof of Theorem 3.3, this is clear if $n \leq m$. For $m<n$, let

$$
F_{m}^{\alpha}(w)=w^{\lfloor(n-1) / 2\rfloor-\lfloor m / 2\rfloor} f_{m}^{\alpha}(w) .
$$

Noting that $\lfloor n / 2\rfloor=\epsilon+\lfloor(n-1) / 2\rfloor$, we see that the left-hand side of (5.1) is the value at $w=\alpha_{n}$ of

$$
w^{\epsilon}\left(F_{m}^{\alpha}(w)-\pi_{n}^{\alpha}\left(F_{m}^{\alpha}\right)(w)\right)
$$

where $\pi_{n}^{\alpha}$ is as defined in the proof of Theorem 3.3. However, it is clear that $F_{m}^{\alpha}(w)$ has degree less than $n$, so it is fixed by $\pi_{n}^{\alpha}$ and (5.1) holds for $m<n$.

The following examples of stable operations for periodic $K$-theory fit into this context.

(5.1) Let $R=\left\{f(w) \in \mathbb{Q}\left[w, w^{-1}\right] \mid f\left(\mathbb{Z}_{(p)}^{\times}\right) \subseteq \mathbb{Z}_{(p)}\right\} \cong K_{(p)_{0}}\left(K_{(p)}\right)$. Then $R \cap$ $\mathbb{Q}[w]=\operatorname{Int}\left(\mathbb{Z}_{(p)}^{\times}, \mathbb{Z}_{(p)}\right) \cong K_{(p)_{0}}\left(k_{(p)}\right)$. For $p$ odd and $q$ primitive $\bmod p^{2}$, taking $\alpha_{i}=q^{i}$ in Theorem 2.3 as in example (4.2) gives a basis for $R \cap \mathbb{Q}[w]$, and then Theorem 5.2 provides a topological basis for $R^{*} \cong K_{(p)}^{0}\left(K_{(p)}\right)$. Up to unit multiples, this recovers the basis given in [10, Theorem 6.2].

For $p=2$, the same procedure carried out with the sequence $\alpha_{i}=$ $(-1)^{i} 3^{\lfloor i / 2\rfloor}$ yields a topological basis for $K_{(2)}^{0}\left(K_{(2)}\right)$. This basis is new-the periodic 2-local case was omitted from [10].

(5.2) Let $R=\left\{f(z) \in \mathbb{Q}\left[z, z^{-1}\right] \mid f\left(1+p \mathbb{Z}_{(p)}\right) \subseteq \mathbb{Z}_{(p)}\right\} \cong G_{0}(G)$. Then, as in example (4.3), $R \cap \mathbb{Q}[w]=\operatorname{Int}\left(1+p \mathbb{Z}_{(p)}, \mathbb{Z}_{(p)}\right) \cong G_{0}(g)$ has $\mathbb{Z}_{(p)}$-basis the sequence of polynomials corresponding to $\alpha_{i}=\hat{q}^{i}$ and, up to unit multiples, Theorem [5.2 recovers the topological basis for $G^{0}(G)$ given in [10, Theorem 6.13].

The $p=2$ variant corresponds to the sequence $\alpha_{i}=9^{i}$ and yields a topological basis for $K O_{(2)}^{0}\left(K O_{(2)}\right)$.

\section{Summary of EXAmPles}

We give a table summarizing our list of examples. To include the periodic examples from Section 5, we introduce some notation for integer-valued Laurent polynomials. Let

$$
\operatorname{IntL}\left(S, \mathbb{Z}_{(p)}\right)=\left\{f(w) \in \mathbb{Q}\left[w, w^{-1}\right] \mid f(S) \subseteq \mathbb{Z}_{(p)}\right\},
$$

the ring of Laurent polynomials that are $p$-locally integer-valued on $S \subseteq \mathbb{Z}_{(p)}$.

For each example, we give the integer sequence $\alpha=\left(\alpha_{i}\right)_{i \geq 0}$, the ring of integervalued polynomials (or Laurent polynomials) for which the $f_{n}^{\alpha}(w)$ (or, respectively, the $\left.w^{-\lfloor n / 2\rfloor} f_{n}^{\alpha}(w)\right)$ provide a basis, and the interpretation of this ring and its dual in terms of $K$-theory (co)operations. 


\begin{tabular}{|cccc|}
\hline$\alpha_{i}$ & $\begin{array}{c}\text { Integer-valued } \\
\text { polynomials }\end{array}$ & $\begin{array}{c}K \text {-theory } \\
\text { cooperations }\end{array}$ & $\begin{array}{c}\text { Dual } K \text {-theory } \\
\text { operations }\end{array}$ \\
\hline$i$ & $\operatorname{Int}(\mathbb{Z}, \mathbb{Z})$ & $Q K_{0}\left(\underline{K}_{0}\right)$ & $P K^{0}\left(\underline{K}_{0}\right)$ \\
$q^{i}$ & $\operatorname{Int}\left(\mathbb{Z}_{(p)}^{\times}, \mathbb{Z}_{(p)}\right)$ & $K_{(p)_{0}}\left(k_{(p)}\right)$ & $k_{(p)}{ }^{0}\left(k_{(p)}\right)$ \\
$q^{i}$ & $\operatorname{IntL}\left(\mathbb{Z}_{(p)}^{\times}, \mathbb{Z}_{(p)}\right)$ & $K_{(p)_{0}}\left(K_{(p)}\right)$ & $K_{(p)}{ }^{0}\left(K_{(p)}\right)$ \\
$(-1)^{i} 3^{\lfloor i / 2\rfloor}$ & $\operatorname{Int}\left(\mathbb{Z}_{(2)}^{\times}, \mathbb{Z}_{(2)}\right)$ & $K_{(2)_{0}}\left(k_{(2)}\right)$ & $k_{(2)}{ }^{0}\left(k_{(2)}\right)$ \\
$(-1)^{i} 3^{\lfloor i / 2\rfloor}$ & $\operatorname{IntL}\left(\mathbb{Z}_{(2)}^{\times}, \mathbb{Z}_{(2)}\right)$ & $K_{(2)_{0}}\left(K_{(2)}\right)$ & $K_{(2)}{ }^{0}\left(K_{(2)}\right)$ \\
$\hat{q}^{i}$ & $\operatorname{Int}\left(1+p \mathbb{Z}_{(p)}, \mathbb{Z}_{(p)}\right)$ & $G_{0}(g)$ & $g^{0}(g)$ \\
$\hat{q}^{i}$ & $\operatorname{IntL}\left(1+p \mathbb{Z}_{(p)}, \mathbb{Z}_{(p)}\right)$ & $G_{0}(G)$ & $G^{0}(G)$ \\
$9^{i}$ & $\operatorname{Int}\left(\left(\mathbb{Z}_{(2)}^{\times}\right)^{2}, \mathbb{Z}_{(2)}\right)$ & $K O_{(2)}\left(k o_{(2)}\right)$ & $k o_{(2)}{ }^{0}\left(k o_{(2)}\right)$ \\
$9^{i}$ & $\operatorname{IntL}\left(\left(\mathbb{Z}_{(2)}^{\times}\right)^{2}, \mathbb{Z}_{(2)}\right)$ & $K O_{(2)_{0}}\left(K O_{(2)}\right)$ & $K O_{(2)}{ }^{0}\left(K O_{(2)}\right)$ \\
$p^{j} q^{m-1-\left\lfloor\frac{m}{p}\right\rfloor}$ & $\operatorname{Int}\left(\mathbb{Z}_{(p)}, \mathbb{Z}_{(p)}\right)$ & $Q K_{(p)_{0}}\left(\underline{K}_{(p)}\right)$ & $P K_{(p)}{ }^{0}\left(K_{(p)}\right)$ \\
$\hat{p}^{j} \hat{q}^{m-1-\left\lfloor\frac{m}{p}\right\rfloor}$ & $\operatorname{Int}\left(\mathbb{Z}_{(p)}^{p-1}, \mathbb{Z}_{(p)}\right)$ & $Q G_{0}\left(\underline{G}_{0}\right)$ & $P G^{0}\left(\underline{G}_{0}\right)$ \\
\hline
\end{tabular}

In the table $p$ is an odd prime, $q$ is primitive $\bmod p^{2}, \hat{p}=p^{p-1}$ and $\hat{q}=q^{p-1}$. In the final two lines $j$ and $m$ are such that $i=p^{j} m>0$ with $p \nmid m$.

\section{REFERENCES}

1. J. F. Adams. Lectures on generalised cohomology. Lecture Notes in Mathematics, 99, Springer, Berlin, 1969. MR0251716 (40:4943)

2. J. F. Adams and F. W. Clarke. Stable operations on complex K-theory. Illinois J. Math. 21 (1977), 826-829. MR0454977 (56:13219)

3. M. Bhargava. P-orderings and polynomial functions on arbitrary subsets of Dedekind rings. J. Reine Angew. Math. 490 (1997), 101-127. MR1468927 (98j:13016)

4. M. Bhargava. The factorial function and generalizations. Amer. Math. Monthly 107 (2000), 783-799. MR.1792411 (2002d:05002)

5. J. M. Boardman. Stable operations in generalized cohomology. Handbook of Algebraic Topology. Edited by I. M. James. North-Holland, Amsterdam, 1995, 585-686. MR.1361899 (97b:55021)

6. J. M. Boardman, D.C. Johnson and W.S. Wilson. Unstable operations in generalized cohomology. Handbook of Algebraic Topology. Edited by I. M. James. North-Holland, Amsterdam, 1995, 687-828. MR1361900 (97b:55022)

7. P.-J. Cahen and J.-L. Chabert. Integer-valued polynomials. Mathematical Surveys and Monographs, 48. American Mathematical Society, Providence, RI, 1997. MR.1421321 (98a:13002)

8. F. Clarke. Self-maps of BU. Math. Proc. Cambridge Philos. Soc. 89 (1981), 491-500. MR602302 (82f:55007)

9. F. Clarke, M. D. Crossley and S. Whitehouse. Bases for cooperations in K-theory. $K$-theory 23 (2001), 237-250. MR.1857208 (2002h:55019)

10. F. Clarke, M. Crossley and S. Whitehouse. Algebras of operations in K-theory. Topology 44 (2005), 151-174. MR2104006 (2006b:55015)

11. E. Helsmoortel, Module de continuité de polynômes d'interpolation. Applications à l'étude du comportement local des fonctions continues sur un compact régulier d'un corps local. C. R. Acad. Sci. Paris 268 (1969), 1168-1171. MR0244212 (39:5529)

12. K. Johnson, The invariant subalgebra and anti-invariant submodule of $K_{*} K_{(p)}$. J. $K$-Theory 2 (2008), no. 1, 123-145. MR2434169 (2009g:55026)

13. L. Schwartz. Opérations d'Adams en K-homologie et applications. Bull. Soc. Math. France 109 (1981), 237-257. MR623792 (82j:55006) 
14. M.-J. Strong. Additive unstable operations in complex K-theory and cobordism. Ph.D. thesis, Department of Pure Mathematics, University of Sheffield, September 2008.

15. M.-J. Strong and Sarah Whitehouse. Infinite sums of unstable Adams operations and cobordism, arXiv:0812.1481v1, to appear in J. Pure Appl. Alg.

Department of Pure Mathematics, University of Sheffield, Sheffield S3 7RH, United KINGDOM

Department of Pure Mathematics, University of Sheffield, Sheffield S3 7RH, United KINGDOM

E-mail address: s.whitehouse@sheffield.ac.uk 